\title{
Influence of pelvic incidence-lumbar lordosis mismatch on surgical outcomes of short-segment transforaminal lumbar interbody fusion
}

Yasuchika Aoki ${ }^{14^{*}}$, Arata Nakajima ${ }^{2}$, Hiroshi Takahashi ${ }^{2}$, Masato Sonobe $^{2}$, Fumiaki Terajima ${ }^{2}$, Masahiko Saito ${ }^{2}$, Kazuhisa Takahashi ${ }^{3}$, Seiji Ohtori ${ }^{3}$, Atsuya Watanabe ${ }^{1,4}$, Takayuki Nakajima ${ }^{1,4}$, Makoto Takazawa ${ }^{1,4}$, Sumihisa Orita ${ }^{3}$, Yawara Eguchi ${ }^{5}$ and Koichi Nakagawa ${ }^{2}$

\begin{abstract}
Background: The importance of pelvic incidence-lumbar lordosis (PI-LL: PI minus LL) mismatch is emphasized in long-segment fusion for adult spinal deformity; however, there are few studies evaluating the influence of PI-LL on surgical outcomes after short-segment fusion. In this study, we have examined the effects of PI-LL mismatch on surgical outcomes of short-segment lumbar intervertebral fusion for lumbar degenerative diseases.

Methods: Patients with lumbar degenerative disease treated by short-segment ( 1 or 2 levels) transforaminal lumbar interbody fusion were divided into Group A (PI-LL $\left.\leq 10^{\circ}: n=22\right)$ and Group B (PI-LL $\geq 11^{\circ}: n=30$ ). Pre-and post-operative patient symptoms were assessed by the visual analogue scale (VAS: scores 0-100 mm; for LBP, lower-extremity pain, and lower-extremity numbness), a detailed VAS for LBP while in motion, standing, and sitting, and the Oswestry disability index (ODI). Surgical outcomes were evaluated by the Nakai score $(3=$ excellent to $0=$ poor. Post-operative data were acquired for at least one year following surgery and were compared between the two groups. Multiple regression analyses were used to evaluate the relative influence of PI-LL on each pre-and post-operative parameter (VAS, detailed VAS and ODI) adjusted for age, sex, fusion levels, body mass index, presence of scoliosis, diabetes mellitus and depression.

Results: The surgical outcomes in Group A were significantly better than those of Group B. Group A showed better post-operative VAS scores for LBP, particularly LBP while standing (11.9 vs. 25.8). The results of the multivariate analyses showed no significant correlation between PI-LL and pre-operative symptoms, but did show a significant correlation between PI-LL and the post-operative VAS score for LBP, lower extremity pain, and numbness.

Conclusions: This study is the first to find that PI-LL mismatch influences post-operative residual symptoms, such as LBP, lower extremity pain and numbness. Among the three types of LBP examined in the detailed VAS, LBP while standing was most strongly related to PI-LL mismatch. The importance of maintaining spinopelvic alignment is emphasized, particularly when treating patients with adult spinal deformity using long-segment fusion surgery. However, our results indicate that surgeons should pay attention to sagittal spinopelvic alignment and avoid post-operative PI-LL mismatch even when treating patients with short-segment lumbar interbody fusion.
\end{abstract}

Keywords: Sagittal alignment, Spinopelvic, Flat-back, Pelvic incidence, Lumbar lordosis, Lumbar interbody fusion

\footnotetext{
* Correspondence: yasuaoki35@fc4.so-net.ne.jp

${ }^{1}$ Department of Orthopaedic Surgery, Eastern Chiba Medical Center, 3-6-2

Okayamadai, Togane, Chiba 283-8686, Japan

${ }^{4}$ Department of General Medical Science, Graduate School of Medicine,

Chiba University, 1-8-1 Inohana,Chuo-ku, Chiba city, Chiba 260-8670, Japan

Full list of author information is available at the end of the article
} 


\section{Background}

Transforaminal lumbar interbody fusion (TLIF) is commonly used to treat patients with lumbar degenerative disease [1]. TLIF offers the advantage of obtaining a circumferential arthrodesis through a unilateral approach with minimal retraction of neural elements. Many surgeons have reported good clinical and radiological outcomes for such lumbar interbody fusion techniques. [2-5] However, there are some patients who have postoperative residual, lower extremity pain, or numbness even without post-operative complications [2-5].

Loss of lordosis has been thought to be one of the factors influencing post-operative residual symptoms $[6,7]$. In 2010, Schwab et al., reported the importance of maintaining global sagittal alignment when performing longsegment spinal fusion surgery in the treatment of adult spinal deformity [8]. Their results clearly showed that post-operative global sagittal balance is significantly correlated with post-operative residual pain and disability [8]. Radiological parameters that most highly correlate with pain and disability are sagittal vertical axis (SVA), pelvic tilt (PT), and the balance of pelvic incidence (PI) and lumbar lordosis (LL) [9]. Schwab et al. proposed radiological parameter thresholds predictive of worse clinical symptoms and poorer quality of life, and concluded that a PI-LL (PI minus LL) of $10^{\circ}$ or less was the ideal spinopelvic alignment for reducing postoperative pain and disability [9]. Therefore, surgeons should plan corrective surgeries to acquire an adequate LL that achieves a harmonious spinopelvic alignment (PI-LL $10^{\circ}$ or less).

In previous studies [8-10], the importance of maintaining appropriate PI-LL has been emphasized, particularly for long-segment fusion for adult spinal deformity; however, there are few studies evaluating the influence of PILL on surgical outcomes following short-segment fusion. In this study, we examined the effect of PI-LL mismatch on surgical outcomes of short-segment TLIF for lumbar degenerative diseases.

\section{Methods}

Patients with lumbar degenerative diseases, such as lumbar spinal stenosis, degenerative spondylolisthesis and spondylolytic spondylolisthesis, treated by shortsegment 1 or 2 level TLIF were included in the present study (Table 1). Patients who showed any indication of other pathological problems, such as vertebral fractures, tumors, or infectious diseases, were excluded. Fifty-two consecutive patients meeting the criteria were divided into Group A (PI-LL $\leq 10^{\circ}: n=22$ ) and Group B (PI-LL $\geq 11^{\circ} ; n=30$ ) by post-operative radiological evaluation.

Pre-operative characteristics of patients, including age, sex, number of fusion levels, body mass index
Table 1 Fusion levels and indications for fusion in groups $A$ and $B$

\begin{tabular}{|c|c|c|c|}
\hline & & Group A $(n=22)$ & Group B $(n=30)$ \\
\hline \multicolumn{2}{|l|}{$\begin{array}{l}\text { Number of } \\
\text { fusion levels }\end{array}$} & $1.1 \pm 0.4$ & $1.4 \pm 0.5^{\mathrm{a}}$ \\
\hline Level of fusion & (No. of cases) & & \\
\hline \multirow[t]{3}{*}{ One level } & L3-L4 & 0 & 1 \\
\hline & L4-L5 & 16 & 12 \\
\hline & L5-S1 & 4 & 5 \\
\hline \multirow[t]{2}{*}{ Two levels } & L3-L4-L5 & 1 & 9 \\
\hline & L4-L5-S1 & 1 & 3 \\
\hline \multicolumn{4}{|c|}{ Indications for fusion } \\
\hline \multicolumn{2}{|c|}{ Degenerative spondylolisthesis } & 14 & 19 \\
\hline \multicolumn{2}{|c|}{ Spondylolytic spondylolisthesis } & 2 & 1 \\
\hline \multicolumn{2}{|c|}{ Spinal canal stenosis } & 6 & 10 \\
\hline
\end{tabular}

${ }^{a}$ Statistically significant difference $p<0.05$

(BMI), and incidence of degenerative scoliosis, diabetes mellitus, and depression, were compared between the two groups.

Anteroposterior and lateral radiographs in the neutral standing position were taken pre-and post-operatively. On anteroposterior radiographs, pre-operative scoliotic curvature was evaluated using the Cobb method. On lateral radiographs, PI and LL were evaluated one-year after surgery. The PI is measured as the angle between a line drawn perpendicular to the sacral end plate at its midpoint and a line drawn from the midpoint of the sacral end plate to the midpoint of the femoral head axis. The LL is the sagittal Cobb angle measured between the superior end plate of L1 and the superior end plate of S1 [8]. Post-operative parameters, such as PI, LL, and PI-LL, were then compared between the two groups. Excellent inter- and intra-rater reliability has been reported for these measurements [10].

\section{Evaluation of surgical outcomes and clinical symptoms}

Intra-and post-operative complications were retrospectively reviewed for each group. Surgical outcomes were evaluated using the Nakai score (Table 2: $3=$ excellent to $0=$ poor) [11]. Pre-and post-operative patient symptoms were also assessed by the visual analogue scale (VAS: scores $0-100 \mathrm{~mm}$; for LBP, lower-extremity pain, and lower-extremity numbness) and the Oswestry disability index (ODI). In addition, the detailed VAS scoring system, originally developed by us for LBP while in motion, standing, and sitting, was used for a more detailed evaluation of LBP, pre-and post-operatively [12]. Postoperative data were prospectively acquired for at least one year following surgery. All scores were compared between the two groups. 
Table 2 Nakai score

\begin{tabular}{l}
\hline Scoring used in the study \\
\hline The patient has resumed work-related and other activities with slight or no symptoms. \\
The patient has resumed work-related and other activities but occasionally feels pain in the back or lower limbs after strenuous work. \\
$\begin{array}{l}\text { The patient has reduced work-related and other activities because of residual pain in the back or lower limbs. } \\
\text { The patient cannot work or carry out activities of daily living and is considered to be disabled. }\end{array}$ \\
\hline
\end{tabular}

\section{Surgical procedures}

All patients underwent surgery in the prone position after induction of general endotracheal anesthesia. The side selected for the unilateral approach was the side showing more neurological involvement. If symptoms were equal on both sides, the approach was made on the left side. A midline incision exposed the posterior elements and the lateral aspect of the facet joint. Following ipsilateral laminotomy, partial facetectomy exposed the surface of the intervertebral disc. Resection of the bone was performed manually using an osteotome and Kerrison rongeur to preserve local bone. Following the removal of disc material and meticulous endplate preparation, the resected local bone was milled and packed into the intervertebral disc space as a bone graft. One or two fusion cages packed with milled local bone were then inserted into the disc space. Contralateral decompression was performed if needed; the contralateral ligamentum flavum, ventral aspect of the lamina, and medial aspect of the contralateral facet joints were resected through the ipsilateral side. Following neural decompression, pedicle screws were placed on both sides. A 1-cm fascia incision allowed insertion of pedicle screws transfascially, using a percutaneous pedicle screw system. Adequate compressive force by the pedicle screws was applied to the disc space to establish stability of the fusion cages.

The percutaneus pedicle screws used for the patients in this series were Viper (Depuy Synthes Spine, Raynham, MA) or Mantis (Stryker, Kalamazoo, MI).

Written informed consent was obtained prior to surgery. The study protocol was approved by the institutional ethics committee of Toho University Sakura Medical Center (No. 2012-071).

\section{Statistical analysis}

Statistical analyses used were the unpaired t-test for age, fusion levels, BMI, PI, LL, PI-LL and VAS, the Mann-Whitney U test for the Nakai score and ODI, and the chi-square test for sex and incidence of each disease.

Multiple regression analysis was performed to investigate the relative influences of pre-and post-operative PILL on each pre-and post-operative VAS, detailed VAS, and ODI after adjustment for age, sex, fusion levels, BMI, presence of scoliosis, diabetes mellitus and depression. PI-LL was utilized as the independent variable and pre-and post-operative data for VAS and ODI as the dependent variables.
Probability values of $p<0.05$ were considered significant. Values are expressed as the mean \pm standard deviation (SD).

\section{Results}

Patient characteristics of Group A (mean age: 67.9 years; range: 44-83 years: 14 male and 8 female) and Group B (mean age: 65.6 years; range: $35-86$ years: 11 male and 19 female), are presented in Table 3. No significant differences were observed between the two groups, except in the post-operative radiological evaluations. Significant differences between groups were found in post-operative PI, LL, and PI-LL (Table 3). The post-operative LL (Group A: 44.0 \pm 8.3 ; Group B: $29.4 \pm 10.7$ ) was not significantly different from the pre-operative LL (Group A: $43.1 \pm 11.4$; Group B: $30.4 \pm 12.4$ ) in both groups. As described in Table 1, the number of fusion levels differed significantly between the two groups.

\section{Surgical complications}

In Group A, one patient had a pulmonary embolism, but recovered with anticoagulant therapy, and one patient had a post-operative epidural hematoma that required additional surgery (Table 4). In Group B, one patient had post-operative cage migration, one patient had pedicle

Table 3 Characteristics of patients in groups A and B

\begin{tabular}{|c|c|c|c|c|}
\hline & & $\begin{array}{l}\text { Group A } \\
(\leq 10 \text { deg.; } n=22)\end{array}$ & $\begin{array}{l}\text { Group B } \\
(\geq 11 \text { deg.; } n=30)\end{array}$ & $p$ value \\
\hline Age & (years) & $67.9 \pm 8.3$ & $67.5 \pm 11.8$ & NS \\
\hline Sex & $(M / F)$ & $14 / 8$ & $11 / 19$ & NS \\
\hline $\begin{array}{l}\text { Body mass } \\
\text { index }\end{array}$ & $\left(\mathrm{kg} / \mathrm{m}^{2}\right)$ & $23.7 \pm 2.6$ & $25.4 \pm 3.9$ & NS \\
\hline $\begin{array}{l}\text { Degenerative } \\
\text { scoliosis }\end{array}$ & ( $\geq 10$ deg) & $22.7 \%(5 / 22)$ & $40.0 \%(12 / 30)$ & NS \\
\hline $\begin{array}{l}\text { Diabetes } \\
\text { mellitus }\end{array}$ & & $0.0 \%(0 / 22)$ & $10.0 \%(3 / 30)$ & NS \\
\hline Depression & & $4.5 \%(1 / 22)$ & $6.7 \%(2 / 30)$ & NS \\
\hline $\begin{array}{l}\text { Pelvic } \\
\text { incidence (PI) }\end{array}$ & (Degree) & $44.9 \pm 7.0$ & $52.1 \pm 11.7$ & $p=0.013$ \\
\hline $\begin{array}{l}\text { Lumbar } \\
\text { lordosis (LL) }\end{array}$ & (Degree) & $44.0 \pm 8.3$ & $29.4 \pm 10.7$ & $p<0.001$ \\
\hline $\begin{array}{l}\mathrm{PI} \text { minus LL } \\
(\mathrm{PI}-\mathrm{LL})^{\mathrm{a}}\end{array}$ & (Degree) & $1.0 \pm 5.8$ & $22.7 \pm 8.9$ & $p<0.001$ \\
\hline Follow-up & (months) & $16.9 \pm 6.6$ & $15.9 \pm 5.3$ & NS \\
\hline
\end{tabular}

apostoperative data; NS is non-significant at $p>0.05$ 
Table 4 Complications during the 1-year minimum follow-up

\begin{tabular}{lll}
\hline Cage migration & $\begin{array}{l}\text { Group A no. of } \\
\text { cases (\%) }\end{array}$ & $\begin{array}{l}\text { Group B no. of } \\
\text { cases (\%) }\end{array}$ \\
\hline Screw malposition & $0(0.0)$ & $1(3.3)$ \\
Pulmonary embolism & $0(0.0)$ & $1^{\mathrm{a}}(3.3)$ \\
Dural tear & $1(4.5)$ & $0(0.0)$ \\
Infection & $0(0.0)$ & $2^{\mathrm{b}}(6.6)$ \\
Postoperative epidural hematoma & $1^{\mathrm{a}}(4.5)$ & $0(0.0)$ \\
Blood transfusion & $0(0.0)$ & $0(0.0)$ \\
Re-operation & $1(4.5)$ & $1(0.0)$ \\
\hline
\end{tabular}

${ }^{a}$ Cases requiring additional surgery to correct complications. ${ }^{b}$ Dural sutures required

screw malposition that required corrective surgery and two patients had dural tears that required dural sutures (Table 4). The incidence of surgical complication did not differ significantly between the two groups. No long-term negative effects continued until the final follow-up in any case with a surgical complication. Homologous blood transfusions were not required in either group.

\section{Comparisons of the Nakai score, pre-and post-operative VAS scores, and ODI}

Surgical outcomes and post-operative residual symptoms evaluated at $16.9 \pm 6.6(12-32)$ months in Group A, and $15.9 \pm 5.3(12-29)$ months in Group B, did not differ significantly between the two groups. In Group A, all 22 patients had good or excellent surgical outcomes, compared to $80.0 \%(24 / 30)$ in Group B. The Nakai scores converted to $0=$ poor to $3=$ excellent were compared between the two groups. Group A exhibited a significantly better score than Group B $(p=0.008)$ (Table 5).

In VAS scores for LBP, Group A showed a better score, both pre- and post-operatively, with a significant difference pre-operatively $(p=0.046)$, and a nonsignificant tendency post-operatively $(p=0.096)$ (Table 5$)$. No significant difference was found in pre-and postoperative VAS scores for lower extremity pain and numbness between the two groups (Table 5).

The ODI showed no significant differences between the two groups pre-and post-operatively (Table 5).

A more thorough analysis of LBP using the detailed VAS scoring system revealed that both groups showed similar pre-operative levels of LBP while standing, but post-operatively, Group A showed significantly less LBP while standing than Group B $(p=0.039)$ (Table 5). In both pre-and post-operative data, no significant differences were found in LBP while in motion and while sitting between the two groups (Table 5).
Table 5 Comparisons of pre-and post-operative scores for the visual analogue scale (VAS), Oswestry disability index (ODI), and detailed VAS for low back pain while in motion, standing, and sitting in groups $A$ and $B$

\begin{tabular}{lllll}
\hline & & Group A & Group B & $p$ value \\
\hline Nakai score & & $2.59 \pm 0.50$ & $2.07 \pm 0.78$ & $p=0.008$ \\
Low back pain (LBP) & Pre-op & $48.9 \pm 27.5$ & $63.2 \pm 22.6$ & $p=0.046$ \\
& Post-op & $14.1 \pm 19.4$ & $23.8 \pm 20.9$ & NS $(p=0.096)$ \\
Lower extremity pain & Pre-op & $68.2 \pm 24.8$ & $66.9 \pm 25.3$ & NS \\
& Post-op & $13.7 \pm 19.2$ & $20.9 \pm 23.3$ & NS \\
Lower extremity & Pre-op & $62.8 \pm 27.4$ & $64.3 \pm 32.0$ & NS \\
numbness & & & & \\
\multirow{2}{*}{ ODI } & Post-op & $11.7 \pm 17.3$ & $20.2 \pm 26.7$ & NS \\
& Pre-op & $41.3 \pm 15.4$ & $47.4 \pm 18.7$ & NS \\
LBP while in motion & Post-op & $17.1 \pm 13.1$ & $22.0 \pm 15.7$ & NS \\
& Pre-op & $49.1 \pm 35.2$ & $55.6 \pm 32.2$ & NS \\
LBP while standing & Post-op & $19.2 \pm 26.0$ & $22.6 \pm 23.9$ & NS \\
& Pre-op & $63.8 \pm 30.9$ & $73.0 \pm 25.0$ & NS \\
& Post-op & $11.9 \pm 17.6$ & $25.8 \pm 26.7$ & $p=0.039$ \\
LBP while sitting & Pre-op & $37.0 \pm 27.4$ & $43.9 \pm 27.2$ & NS \\
& Post-op & $12.3 \pm 17.3$ & $16.1 \pm 19.4$ & NS
\end{tabular}

Nakai score: $3=$ excellent to $0=$ poor; VAS scores: $0-100 \mathrm{~mm}$ Values are expressed as the mean \pm standard deviation. NS is non-significant at $p>0.05$

\section{Correlation between PI-LL and post-operative symptoms}

A multivariate analysis was performed, adjusted for age, sex, fusion levels, BMI, presence of scoliosis, diabetes mellitus and depression, with pre-and post-operative VAS and ODI as dependent variables, and PI-LL as an independent variable. The results showed no significant association between PI-LL and pre-operative parameters (Table 6). However, greater PI-LL was significantly associated with worse post-operative VAS for LBP $(p=0.045)$, lower extremity pain $(p=0.024)$, and numbness $(p=0.019)$ (Table 7). No significant association was found between PI-LL and post-operative ODI (Table 7). Among the detailed VAS scores in the three situations, a larger PI-LL was significantly associated with worse LBP while standing $(p=0.038)$, but there was no significant association with LBP while in motion or sitting (Table 7).

\section{Discussion}

Post-operative flat-back syndrome has been reported to be a factor causing undesirable surgical outcomes after lumbar fusion surgery $[6,7]$. Schwab et al. recently proposed a systematic evaluation method to classify sagittal spinal alignment in flat-back cases [8]. Their study demonstrated that SVA, PT, and PI-LL were most closely related to LBP and disability. Since the Scoliosis Research Society-Schwab adult spinal deformity classification was established [10], there have been no studies examining 
Table 6 Correlations between PI-LL and pre-operative VAS, detailed VAS, and ODI

\begin{tabular}{|c|c|c|c|c|}
\hline Valuables & $\begin{array}{l}\text { Regression } \\
\text { coefficient }\end{array}$ & $\begin{array}{l}\text { Standardized regression } \\
\text { coefficient }\end{array}$ & t value & $p$ value \\
\hline \multirow[t]{2}{*}{ VAS for LBP } & 0.355 & 0.198 & 1.216 & NS \\
\hline & & & & $p=0.229$ \\
\hline VAS for lower & 0.390 & 0.223 & 1.377 & NS \\
\hline Extremity pain & & & & $p=0.174$ \\
\hline VAS for lower & 0.078 & 0.037 & 0.235 & NS \\
\hline $\begin{array}{l}\text { Extremity } \\
\text { numbness }\end{array}$ & & & & $p=0.815$ \\
\hline \multirow[t]{2}{*}{ ODI } & 0.382 & 0.310 & 1.980 & NS \\
\hline & & & & $p=0.053$ \\
\hline $\begin{array}{l}\text { Detailed } \\
\text { VAS for }\end{array}$ & 0.371 & 0.158 & 0.938 & NS \\
\hline $\begin{array}{l}\text { LBP while in } \\
\text { motion }\end{array}$ & & & & $p=0.353$ \\
\hline $\begin{array}{l}\text { Detailed } \\
\text { VAS for }\end{array}$ & 0.384 & 0.197 & 1.186 & NS \\
\hline $\begin{array}{l}\text { LBP while } \\
\text { standing }\end{array}$ & & & & $p=0.241$ \\
\hline $\begin{array}{l}\text { Detailed } \\
\text { VAS for }\end{array}$ & 0.474 & 0.248 & 1.466 & NS \\
\hline $\begin{array}{l}\text { LBP while } \\
\text { sitting }\end{array}$ & & & & $p=0.149$ \\
\hline
\end{tabular}

NS is non-significant at $p>0.05$

PI pelvic incidence; $L L$ lumbar lordosis; PI-LL PI minus LL; VAS visual analogue scale; $O D I$ Oswestry disability index; $L B P$ low back pain

Table 7 Correlations between PI-LL and post-operative VAS, detailed VAS, and ODI

\begin{tabular}{|c|c|c|c|c|}
\hline Valuables & $\begin{array}{l}\text { Regression } \\
\text { coefficient }\end{array}$ & $\begin{array}{l}\text { Standardized } \\
\text { regression } \\
\text { coefficient }\end{array}$ & t value & $p$ value \\
\hline VAS for LBP & 0.517 & 0.335 & 2.052 & $p=0.045$ \\
\hline $\begin{array}{l}\text { VAS for lower extremity } \\
\text { pain }\end{array}$ & 0.577 & 0.348 & 2.321 & $p=0.024$ \\
\hline $\begin{array}{l}\text { VAS for lower extremity } \\
\text { numbness }\end{array}$ & 0.639 & 0.360 & 2.417 & $p=0.019$ \\
\hline \multirow[t]{2}{*}{ ODI } & 0.241 & 0.215 & 1.372 & NS \\
\hline & & & & $p=0.176$ \\
\hline Detailed VAS for & 0.401 & 0.213 & 1.288 & NS \\
\hline LBP while in motion & & & & $p=0.204$ \\
\hline $\begin{array}{l}\text { Detailed VAS for LBP } \\
\text { while standing }\end{array}$ & 0.626 & 0.341 & 2.134 & $p=0.038$ \\
\hline Detailed VAS for & 0.379 & 0.270 & 1.632 & NS \\
\hline LBP while sitting & & & & $p=0.109$ \\
\hline
\end{tabular}

NS is non-significant at $p>0.05$

$P I$ pelvic incidence; $L L$ lumbar lordosis; $P I-L L$ PI minus LL; VAS visual analogue scale; $O D I$ Oswestry disability index; $L B P$ low back pain the influence of PI-LL mismatch on surgical outcomes after short-segment lumbar interbody fusion. Our study is the first to show that PI-LL mismatch significantly correlated with surgical outcomes after short-segment lumbar interbody fusion. Consequently, our study reveals that surgical outcomes after short-segment lumbar interbody fusion will be poorer when good spinopelvic balance is not achieved following surgery. Thus, surgeons should pay attention to the sagittal spinopelvic balance of patients even when treating patients with short-segment lumbar spinal stenosis or spondylolisthesis. Our results show that postoperative PI-LL mismatch causes greater residual LBP. Interestingly, in this study, our originally developed detailed VAS system was successful in revealing that LBP while standing is most closely correlated with postoperative PI-LL mismatch. Patients with flat-back syndrome are usually characterized by an inability to stand erect and back pain [7]. In our study, post-operative residual LBP while standing may reflect the inability to stand erect due to flat-back syndrome.

We believe that PI-LL did not correlate with preoperative symptoms (Table 6) because pre-operative symptoms, including pain caused by nerve root compression, spinal instability, and spinopelvic alignment, are more complex than post-operative symptoms. Postoperative symptoms, particularly LBP while standing, were more simply influenced by spinopelvic alignment (Table 7), because symptoms caused by nerve root compression and spinal instability may have been relieved by fusion surgery. Therefore, PI-LL significantly correlated with post-operative LBP, but not with preoperative LBP (Tables 6, 7).

There are some limitations to this study. Most importantly, post-operative residual symptoms are affected by multiple factors. In our study, Group A showed patient characteristics that differed from those of Group B. Although most were not significant (Tables 1,3 ), the possibility that differing characteristics influenced the results of the comparison study between Group A and Group B persists. In particular, the difference in number of fusion levels may affect surgical outcomes because better surgical outcomes would be expected from single-level fusion than two-level fusion [13]. However, the multivariate analysis adjusted for age, sex, fusion levels, BMI, presence of scoliosis, diabetes mellitus and depression showed that PILL was significantly associated with post-operative symptoms, not with pre-operative symptoms. These results lead us to conclude that post-operative PI-LL mismatch causes more intense post-operative residual symptoms, particularly LBP while standing. There are other limitations, including the short post-operative follow-up and small number of patients, and the variety of pathologies that were indicators for fusion surgery in the study. There is a concern that short-term outcomes may be influenced by 
surgery-related factors, rather than radiologic factors. However, in our study no patients with surgical complication had deleterious consequences from the complication after additional treatments, such as screw reposition, hematoma removal, or dural suture were accomplished. Recent studies reported that a post-operative PI-LL mismatch resulted in increased load on adjacent segments and predisposition to adjacent segment pathologies and late surgical complications seen after longer follow-ups [14-17]. Thus, longer follow-up times may reveal an effect of adjacent segment pathology or late surgical complications on surgical outcomes. However, the statistically significant differences found between our two study groups, despite the small number of subjects, lead us to believe our results are reliable and provide important information for spine surgeons treating lumbar degenerative disease. Further investigations in which inclusion criteria are stricter with a longer follow-up and larger number of subjects are needed to confirm our conclusions.

In this study, we did not examine the effects of correcting LL on surgical outcomes because the surgery in this series was not intended to correct LL. Therefore, we cannot conclude that the surgical correction of LL would have a favorable effect on surgical outcomes. However, there have been several studies showing the positive effects of improving sagittal spinopelvic alignment on surgical outcomes of short-segment fusion surgery. Kim et al. found that patients with improved PT after posterior lumbar interbody fusion (PLIF) had better clinical results [18]. Lazennec et al. reported that a larger post-operative PT positively correlated with LBP after lumbosacral fusion surgery [19]. A larger PT, which reflects pelvic retroversion, is usually correlated with loss of LL [20]; therefore, we surmise that the surgical correction of LL decreases PT and produces improved surgical outcomes. Hioki et al. found that two-level PLIF provided satisfactory results by preserving lordosis of the lumbar spine, suggesting the importance of restoring LL by fusion surgery [21]. However, Lazennec et al. reported that post-operative loss of lordosis was not always related to post-operative residual symptoms [19]. One explanation for this inconsistency may be that the appropriate LL varies depending on a patient's PI. These findings indicate the importance of acquiring ideal PI-LL when performing short-segment lumbar fusion.

More recently, Kim et al. reported that TLIF has the potential to cause post-operative flat back, and that PLIF is more reliable than TLIF for restoring spinopelvic alignment [22]. For the maintenance of segmental lumbar lordosis, extreme lateral interbody fusion (XLIF) is thought to have more capability to increase disc height and correct spinopelvic alignment [23-25]. From these observations, surgeons can select the appropriate surgical technique from the various fusion methods, TLIF,
PLIF, XLIF, multi-segment fusion, spinal osteotomy, etc., taking into consideration the ability of each method to restore spinopelvic alignment. Efforts should be made to reduce PI-LL to $10^{\circ}$ or less whenever feasible.

\section{Conclusions}

This study is the first to report that PI-LL influences post-operative residual symptoms after short-segment fusion for lumbar degenerative disease. PI-LL mismatch is more closely related to LBP while standing than LBP while in motion or sitting. From these observations, surgeons should be careful to achieve sagittal spinopelvic alignment and avoid post-operative PI-LL mismatch when treating patients with short-segment lumbar interbody fusion, particularly when the patient has severe LBP while standing.

\section{Abbreviations \\ PI: Pelvic incidence; LL: Lumbar lordosis; VAS: Visual analog scale; \\ ODI: Oswestry disability index; LBP: Low back pain; TLIF: Transforaminal lumbar interbody fusion; SVA: Sagittal vertical axis; PT: Pelvic tilt; BMI: Body mass index; PLIF: Posterior lumbar interbody fusion; XLIF: Extreme lateral interbody fusion.}

\section{Competing interests}

The authors declare that they have no competing interests.

\section{Author's contributions}

Author contributions to the manuscript preparation include the following. YA and AN drafted the manuscript, HT, MS, FT, and MS participated in interpretation and acquisition of data, $\mathrm{KT}$ and $\mathrm{SO}$ participated in its design, conception, and helped to draft the manuscript. AW, TN, MT and SO collected patients' information and participated in co-ordination and co-wrote the manuscript. KN participated in its design and statistical analysis. YE participated in data acquisition and manuscript revision. All authors read and approved the final manuscript.

\section{Acknowledgements}

We wish to thank Drs. Shinji Taniguchi and Manabu Yamada for help with data collection.

No financial support was received for this study.

\section{Author details}

${ }^{1}$ Department of Orthopaedic Surgery, Eastern Chiba Medical Center, 3-6-2 Okayamadai, Togane, Chiba 283-8686, Japan. ${ }^{2}$ Department of Orthopaedic Surgery, Toho University Sakura Medical Center, 564-1 Shimoshizu, Sakura, Chiba 285-8741, Japan. ${ }^{3}$ Department of Orthopaedic Surgery, Graduate School of Medicine, Chiba University, 1-8-1 Inohana, Chuoku, Chiba city, Chiba 260-8677, Japan. ${ }^{4}$ Department of General Medical Science, Graduate School of Medicine, Chiba University, 1-8-1 Inohana,Chuo-ku, Chiba city, Chiba 260-8670, Japan. ${ }^{5}$ Department of Orthopaedic Surgery, National Hospital Organization Shimoshizu Hospital, 934-5Shikawatashi, Yotsukaido, Chiba 284-0003, Japan.

Received: 26 March 2015 Accepted: 12 August 2015

Published online: 20 August 2015

\section{References}

1. Harms J, Rolinger H. A one-stage procedure in operative treatment of spondylolisthesis : dorsal traction-reposition and anterior fusion. Z Orthop Ihre Grenzgeb. 1982;120:343-7.

2. Taneichi H, Suda K, Kajino T, Matsumura A, Moridaira H, Kaneda K. Unilateral transforaminal lumbar interbody fusion and bilateral anterior-column fixation with two Brantigan I/F cages per level: clinical outcomes during a minimum 2-year follow-up period. J Neurosurg Spine. 2006;4(3):198-205. 
3. Lauber S, Schulte TL, Liljenqvist U, Halm H, Hackenberg L. Clinical and radiologic 2-4-year results of transforaminal lumbar interbody fusion in degenerative and isthmic spondylolisthesis grades 1 and 2. Spine. 2006;31(15):1693-8.

4. Park P, Foley KT. Minimally invasive transforaminal lumbar interbody fusion with reduction of spondylolisthesis: technique and outcomes after a minimum of 2 years' follow-up. Neurosurg Focus. 2008;25(2):E16. doi:10.3171/FOC/2008/25/8/E16.

5. Aoki Y, Yamagata M, Ikeda Y, Nakajima F, Ohtori S, Nakagawa K, et al. A prospective randomized controlled study comparing transforaminal lumbar interbody fusion techniques for degenerative spondylolisthesis: unilateral pedicle screw and 1 cage versus bilateral pedicle screws and 2 cages. J Neurosurg Spine. 2012;17(2):153-9.

6. Kostuik JP, Maurais GR, Richardson WJ, Okajima Y. Combined single stage anterior and posterior osteotomy for correction of iatrogenic lumbar kyphosis. Spine. 1988;13(3):257-66.

7. La Grone MO. Loss of lumbar lordosis. A complication of spinal fusion for scoliosis. Orthop Clin North Am. 1988;19(2):383-93.

8. Schwab F, Patel A, Ungar B, Farcy JP, Lafage V. Adult spinal deformitypostoperative standing imbalance: how much can you tolerate? An overview of key parameters in assessing alignment and planning corrective surgery. Spine. 2010;35(25):2224-31.

9. Schwab FJ, Blondel B, Bess S, Hostin R, Shaffrey Cl, Smith JS, et al. Radiographical spinopelvic parameters and disability in the setting of adult spinal deformity: a prospective multicenter analysis. Spine. 2013;38(13):E803-12.

10. Schwab F, Ungar B, Blondel B, Buchowski J, Coe J, Deinlein D, et al. Scoliosis Research Society-Schwab adult spinal deformity classification: a validation study. Spine. 2012;37(12):1077-82.

11. Nakai O, Ookawa A, Yamaura I. Long-term roentgenographic and functional changes in patients who were treated with wide fenestration for central lumbar stenosis. J Bone Joint Surg Am. 1991;73(8):1184-91.

12. Aoki Y, Sugiura S, Nakagawa K, Nakajima A, Takahashi H, Ohtori S, et al. Evaluation of nonspecific low back pain using a new detailed visual analogue scale for patients in motion, standing, and sitting: characterizing nonspecific low back pain in elderly patients. Pain Res Treat. 2012;2012:680496. doi:10.1155/2012/680496

13. Sakaura H, Yamashita T, Miwa T, Ohzono K, Ohwada T. Outcomes of 2-level posterior lumbar interbody fusion for 2-level degenerative lumbar spondylolisthesis. J Neurosurg Spine. 2013;19-1:90-4.

14. Senteler M, Weisse B, Snedeker JG, Rothenfluh DA. Pelvic incidence-lumbar lordosis mismatch results in increased segmental joint loads in the unfused and fused lumbar spine. Eur Spine J. 2014;23(7):1384-93.

15. Rothenfluh DA, Mueller DA, Rothenfluh E, Min K. Pelvic incidence-lumbar lordosis mismatch predisposes to adjacent segment disease after lumbar spinal fusion. Eur Spine J. 2014;24(6):1251-8.

16. Reames DL, Kasliwal MK, Smith JS, Hamilton DK, Arlet V, Shaffrey Cl. Time to development, clinical and radiographic characteristics, and management of proximal junctional kyphosis following adult thoracolumbar instrumented fusion for spinal deformity. J Spinal Disord Tech. 2015;28(2):E106-14.

17. Smith JS, Shaffrey E, Klineberg E, Shaffrey Cl, Lafage V, Schwab FJ, et al. Prospective multicenter assessment of risk factors for rod fracture following surgery for adult spinal deformity. J Neurosurg Spine. 2014;21(6):994-1003.

18. Kim MK, Lee SH, Kim ES, Eoh W, Chung SS, Lee CS. The impact of sagittal balance on clinical results after posterior interbody fusion for patients with degenerative spondylolisthesis: a pilot study. BMC Musculoskelet Disord. 2011;12:69. doi:10.1186/1471-2474-12-69.

19. Lazennec JY, Ramare S, Arafati N, Laudet CG, Gorin M, Roger B, et al. Sagittal alignment in lumbosacral fusion: relations between radiological parameters and pain. Eur Spine J. 2000;9-1:47-55.

20. Le Huec JC, Faundez A, Dominguez D, Hoffmeyer P, Aunoble S. Evidence showing the relationship between sagittal balance and clinical outcomes in surgical treatment of degenerative spinal diseases: a literature review. Int Orthop. 2015;39-1:87-95.

21. Hioki A, Miyamoto K, Kodama H, Hosoe H, Nishimoto H, Sakaeda H, et al. Two-level posterior lumbar interbody fusion for degenerative disc disease: improved clinical outcome with restoration of lumbar lordosis. Spine J. 2005:5-6:600-7.

22. Kim JK, Moon BG, Kim DR, Kim JS. Postoperative flat back: contribution of posterior accessed lumbar interbody fusion and spinopelvic parameters. J Korean Neurosurg Soc. 2014;56(4):315-22.
23. Alimi M, Hofstetter CP, Cong GT, Tsiouris AJ, James AR, Paulo D, et al. Radiological and clinical outcomes following extreme lateral interbody fusion. J Neurosurg Spine. 2014;20(6):623-35.

24. Tohmeh A, Khorsand D, Watson B, Zielinski X. Radiographic and clinical evaluation of XLIF: effects of cage and instrumentation type with a minimum of 1 year follow-up. Spine. 2014;39(26):E1582-91.

25. Malham GM, Ellis NJ, Parker RM, Blecher CM, White R, Goss B, et al. Maintenance of segmental lordosis and disc height in standalone and instrumented extreme lateral interbody fusion (XLIF). J Spinal Disord Tech. 2014; Mar 24. [Epub ahead of print]

\section{Submit your next manuscript to BioMed Central and take full advantage of:}

- Convenient online submission

- Thorough peer review

- No space constraints or color figure charges

- Immediate publication on acceptance

- Inclusion in PubMed, CAS, Scopus and Google Scholar

- Research which is freely available for redistribution

Submit your manuscript at www.biomedcentral.com/submit 